\title{
DECIDABILITY OF SECOND-ORDER THEORIES AND AUTOMATA ON INFINITE TREES ${ }^{1}$
}

\author{
BY MICHAEL O. RABIN
}

Communicated by Dana Scott, March 21, 1968

1. Introduction. In this note we announce the solvability of the decision problem of the (monadic) second-order theory of two successor functions (S2S). This answers a question raised by Büchi [1].

The above decidability result turns out to be very powerful in that many difficult, often seemingly unrelated, decision problems are reducible to it. Thus we are able to deduce: the decidability of the first-order theory of the lattice of closed subsets of the real line (in answer to Grzegorczyk [6]); the decidability of the second-order theory of countable linearly ordered sets; decidability of theory of countable Boolean algebras with quantification permitted over ideals; and many other results. All the decidability procedures obtained here are elementary recursive in the sense of Kalmar. Due to the fact that we use reductions to a second-order theory, our decidability proofs are very direct. Through appropriate interpretations, the set variables of S2S allow us to talk about all structures in a certain class.

The method of solution involves the development of a theory of finite automata operating on infinite trees. Complete details will be published elsewhere.

1. Theory of $n$ successor functions. Let $T=\{0,1\}^{*}$ be the set of all finite words on $\{0,1\}$. The functions $r_{0}(x)=x 0, r_{1}(x)=x 1, x \in T$, are called the successor functions. On $T$ define the relation $x \leqq y$ $\equiv \exists z[y=x z] ;$ and the lexicographic total ordering $x \preceq y \equiv x \leqq y$ $\vee \exists z \exists u \exists v[x=z 0 u \wedge y=z 1 v]$.

Let $\Lambda$ denote the empty sequence. A path $\pi$ of $T$ is a subset $\pi \subset T$ such that (1) $\Lambda \in \pi$; (2) for each $x \in \pi$, either $x 0 \in \pi$ or $x 1 \in \pi$; (3) for each $\Lambda \neq x \in \pi$, the predecessor node $y$ of $x$ is in $\pi$.

For $\mathfrak{M}$ a structure and $L$ a formal language, $\operatorname{Th}(\mathfrak{M}, L)$ will denote the theory of $\mathfrak{M}$ in the language $L$. If $\mathscr{T}$ is a class of similar structures, then $\operatorname{Th}(\mathcal{K}, L)=\bigcap_{\mathfrak{M} \in \mathfrak{K}} \operatorname{Th}(\mathfrak{M}, L)$. If $L$ is (monadic) second-order, then we denote $\operatorname{Th}(\mathfrak{M}, L)$ by $\mathrm{Th}_{2}(\mathfrak{M})$. If $L^{\prime}$ is second-order and the set variables are restricted to range over finite subsets of the domain, then $\operatorname{Th}\left(\mathfrak{M}, L^{\prime}\right)$ is called the weak second-order theory of $\mathfrak{M}$.

\footnotetext{
1 Presented to the American Mathematical Society, July 5, 1967.
} 
$\mathrm{Th}_{2}\left(\mathfrak{R}_{2}\right)$, where $\mathfrak{R}_{2}=\left\langle T, r_{0}, r_{1}, \leqq, \preceq\right\rangle$, is denoted by $\mathrm{S} 2 \mathrm{~S}$ and called the second-order theory of two successor functions. In a similar way we define $\mathrm{S} n \mathrm{~S}$ - the second-order theory of $n$ successor functions, for any $1 \leqq n \leqq \omega$.

THEOREM 1.1. The second-order theory of two successor functions (S2S) is decidable.

By direct interpretations we get

CoROllary 1.2. $\mathrm{S} n \mathrm{~S}$ is decidable for every $1 \leqq n \leqq \omega$.

The proof of the decidability of S2S employs automata on infinite trees in a manner to be explained in $\$ 3$.

2. Applications. Let $\mathfrak{K}_{\leqq}^{\omega}$ be the class of all linearly ordered sets $\langle\bar{A}, \leqq\rangle$ such that $c(\bar{A}) \leqq \omega$.

THEOREM 2.1. $\mathrm{Th}_{2}\left(\varkappa_{\leqq}^{\omega}\right)$, the second-order theory of countable linearly ordered sets, is decidable.

Proof. It is readily seen that for every $\langle\bar{A}, \leqq\rangle \in \mathcal{K}_{\leqq}^{\omega}$ there exists a set $A \subseteq T$ so that $\langle\bar{A}, \leqq\rangle \simeq\langle A, \ldots \mid A\rangle$. This directly implies decidability of $\mathrm{Th}_{2}\left(\mathfrak{K}_{\leqq}^{\omega}\right)$.

The notion of a subset $A \subseteq T$ being finite is definable in S2S by a formula $F \boldsymbol{n}(\boldsymbol{A})$. It follows that $\mathrm{S} 2 \mathrm{~S}$ remains decidable upon inclusion of set variables ranging over finite sets. We get as a corollary the following result of Laüchli [7] which strengthens Ehrenfeucht's result [4]. In contrast with the treatment in [4], [7], we get here elementary recursive decision procedures.

COROLLARY 2.2. The weak second-order theory of linearly ordered sets is decidable.

The following result is related to Büchi's Theorem 1' of [2].

COROLLARY 2.3. The second-order theory of countable well-ordered sets is decidable.

Let $\mathfrak{K}_{f}$ be the class of all structures $\langle A, f\rangle$, where $f: A \rightarrow A$; and $\mathfrak{K}_{f}^{\omega}$ be the class of all $\langle A, f\rangle \in \mathfrak{K}_{f}$ with $c(A) \leqq \omega$.

THEOREM 2.4. $\mathrm{Th}_{2}\left(\mathcal{K}_{f}^{\omega}\right)$, the second-order theory of a unary function with a countable domain, is decidable.

The proof is accomplished by reproducing in $\mathfrak{N}_{2}$, through appropriate definitions, the general structure $\langle A, f\rangle \in \mathcal{K}_{f}^{\omega}$. 
COROLlaRY 2.5. The weak second-order theory of a unary function is decidable.

This is a strengthened version of Ehrenfeucht's result [3], where he announced the decidability of the first-order theory of a unary function.

Let $C D=\{0,1\}^{\omega}$ with product topology. Each path $\pi \subset T$ is the set of all finite initials of a unique element $\phi: \omega \rightarrow\{0,1\}$ of $C D$. Thus, we shall view the paths as elements of $C D$, and sets of paths as subsets of $C D$.

Theorem 2.6. Let $\mathrm{Cl}(\boldsymbol{B}, \boldsymbol{A})$ be $[\boldsymbol{B} \subseteq \boldsymbol{A}] \wedge \mathrm{Path}(\boldsymbol{B})$, and $F_{\sigma}(\boldsymbol{B}, \boldsymbol{A})$ be $\mathrm{F} n(A \cap B) \wedge \mathrm{Path}(B) .\left\{\pi \mid \mathfrak{R}_{2} \vDash \mathrm{Cl}(\pi, A)\right\}$ ranges, with $A \subseteq T$, over all closed subsets of $C D$, and $\left\{\pi \mid \mathfrak{N}_{2} \vDash F_{\sigma}(\pi, A)\right\}$ ranges over all $F_{\sigma}$ subsets of $C D$.

THEOREM 2.7. Let $\subseteq=\langle C D$, $\leqq\rangle$ be Cantor's discontinuum with the usual ordering. Let $L$ be a language appropriate to $(5$ which has (besides the individual variables) set variables, $C_{1}, C_{2}, \cdots$, ranging over closed subsets of $C D$, and set variables $D_{1}, D_{2}, \cdots$, ranging over $F_{\sigma}$ subsets of $C D . \mathrm{Th}(\mathfrak{S}, L)$ is decidable.

The above result carries over from $C D$ to the segment $[0,1]$ with the usual topology and order. This implies an affirmative answer to Grzegorczyk's question [6] whether the first-order theory of the lattice of all closed subsets of the real line is decidable.

Denote the class of all Boolean algebras by $\varkappa_{B}$, and the class of countable Boolean algebras by $\varkappa_{B}^{\omega}$. Let $L_{I}$ be the language appropriate for $\mathfrak{K}_{B}$, which has set variables ranging over ideals of the Boolean algebras.

TheOREM 2.8. $\mathrm{Th}\left(\mathcal{K}_{B}^{\omega}, L_{I}\right)$, the theory of countable Boolean algebras with quantification over ideals, is decidable.

This follows from Theorem 2.7 and the fact that $C D$ is the Stone space of the free Boolean algebra with a denumerable number of generators.

As a corollary we get the following improvement of Tarski's result [8]; and of Ershov's result [5, Theorem 9] to the effect that the firstorder theory of Boolean algebras with a distinguished maximal ideal is decidable.

THEOREM 2.9. The first-order theory of Boolean algebras with a sequence of distinguished ideals is decidable. 
3. Automata on infinite trees. For a mapping $\phi: A \rightarrow B$, define $\operatorname{In}(\phi)=\left\{b \mid b \in B, c\left(\phi^{-1}(b)\right) \geqq \omega\right\}$. In the following, $\Sigma$ denotes a finite set called the alphabet.

Definition. A $\Sigma$-(valued) tree is a pair $(v, T)$ such that $v: T \rightarrow \Sigma$. The set of all $\Sigma$-trees will be denoted by $V_{\Sigma}$.

Definition. A $\Sigma$-automaton is a system $\mathfrak{A}=\left\langle S, M, S_{0}, F\right\rangle$ where $S$ is a finite set; $M: S \times \Sigma \rightarrow P(S \times S)(P(A)$ denotes the set of all subsets of $A) ; S_{0} \subseteq S$; and $F \subseteq P(S)$.

Definition. A run of $\mathfrak{A}$ on the $\Sigma$-tree $t=(v, T)$ is a mapping $r: T \rightarrow S$ such that for $y \in T,(r(y 0), r(y 1)) \in M(r(y), v(y))$.

The automaton $\mathfrak{A}$ accepts $t$ if there exists an $\mathfrak{A}$-run $r$ on $t$ such that $r(\Lambda) \in S_{0}$, and for every path $\pi$ of $T, \operatorname{In}(r \mid \pi) \in F$. The set $T(\mathfrak{A})$ of $\boldsymbol{\Sigma}$-trees defined by $\mathfrak{A}$ is $T(\mathfrak{H})=\left\{t \mid t \in V_{\Sigma}, t\right.$ accepted by $\left.\mathfrak{A}\right\}$. A set $A \subseteq V_{\Sigma}$ is automaton definable if for some $\mathfrak{A}, A=T(\mathfrak{R})$.

Let $t=(v, T)$ be a $\Sigma \times \Sigma_{1}$-tree and let $p(x, y)=x$. The projection $p(t)$, by definition, is the $\Sigma$-tree $(p v, T)$.

TheOREM 3.1. If $A, B \subseteq V_{\Sigma}$ and $C \subseteq V_{\Sigma \times \Sigma_{1}}$ are automaton definable, then so are $A \cup B, V_{\Sigma}-A$, and $p(C)$. Automata defining the latter sets can be effectively obtained from automata defining $A, B$ and $C$.

THEOREM 3.2. There exists an effective (even elementary-recursive) procedure for deciding for every automaton $\mathfrak{A}$ whether $T(\mathfrak{H})=\varnothing$.

For a set $A \subseteq T$, let $\chi_{A}: T \rightarrow\{0,1\}$ be the characteristic function of $A$. Denote $\{0,1\}^{n}$ by $\Sigma^{n}, n<\omega$. With $\vec{A}=\left(A_{1}, \cdots, A_{n}\right)$, associate the $\Sigma^{n}$-tree $\left(v_{\vec{A}}, T\right)$ defined by $v_{\vec{A}}(x)=\left(\chi_{A_{1}}(x), \cdots, \chi_{A_{n}}(x)\right), x \in T$. The mapping $\tau: \vec{A} \rightarrow\left(v_{\vec{A}}, T\right)$ sets up a one-to-one correspondence between $P(T)^{n}$ and $V_{\Sigma^{n}}$.

THEOREM 3.3. There exists an (elementary recursive) effective procedure for assigning to every formula $F\left(\boldsymbol{A}_{1}, \cdots, \boldsymbol{A}_{n}\right)$ of $\mathrm{S} 2 \mathrm{~S}$ a $\boldsymbol{\Sigma}^{n_{-}}$ automaton $\mathfrak{A}_{F}$ so that

$$
T\left(\mathfrak{A}_{F}\right)=\tau\left(\left\{\left(A_{1}, \cdots, A_{n}\right) \mid \mathfrak{N}_{2} \vDash F\left(A_{1}, \cdots, A_{n}\right)\right\}\right) .
$$

The combination of Theorems 3.2 and 3.3 at once implies the decidability of S2S. In fact, Theorem 3.3 gives us a complete picture of the relations definable in S2S. Through the interpretations used, we also get information about definability in all the theories proved decidable in $\$ 2$.

\section{BIBLIOGRAPHY}

1. J. R. Büchi, On a decision method in restricted second-order arithmetic, Proc. Internat. Congress Logic, Method. and Philos. Sci. 1960, Stanford Univ. Press, Stanford, Calif., 1962, pp. 1-11. 
2. - Decision methods in the theory of ordinals, Bull. Amer. Math. Soc. 71 (1965), 767-770.

3. A. Ehrenfeucht, Decidability of the theory of one function, Notices Amer. Math. Soc. 6 (1959), 268.

4. - Decidability of the theory of one linear ordering relation, Notices Amer. Math. Soc. 6 (1959), 268-269.

5. Yu. L. Ershov, Decidability of the theory of relatively complemented distributive lattices and the theory of filters, Algebra i. Logika Sem. 3 (1964), 5-12.

6. A. Grzegorczyk, Undecidability of some topological theories, Fund. Math. 38 (1951), 137-152.

7. H. Laüchli, Decidability of the weak second-order theory of linear ordering, Kolloquium über Logik Grundlagen der Math., Hanover 1966 (to appear).

8. A. Tarski, Arithmetical classes and types of Boolean algebras, Bull. Amer. Math. Soc. 55 (1949), 64.

ibM Watson Research Center, Yorktown Heights, and

The Hebrew University, Jerusalem 